\title{
Menisco Lateral Hipermóvil: Diagnóstico y Tratamiento de Lesiones de Fascículos Poplíteo Meniscales
}

\section{Hypermobile Lateral Meniscus: Diagnosis and Treatment of Popliteomeniscal Fasciculi Injuries}

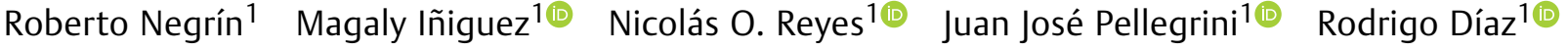 \\ ${ }^{1}$ Departamento de Traumatología y Ortopedia, Clínica Las Condes, \\ Santiago, Chile \\ Address for correspondence Roberto Negrín, MD, Department of \\ Traumatology and Orthopedics, Clínica Las Condes, Estoril 450, Las \\ Condes, Santiago, Chile (e-mail: rnegrin@clc.cl).
} Rev Chil Ortop Traumatol 2019;60:91-96.

\section{Resumen \\ Palabras clave \\ - rodilla \\ - hipermóvil menisco lateral \\ - romperse la rodilla}

\section{Abstract}

received

March 21, 2019

accepted

September 9, 2019
El Síndrome de Menisco Hipermóvil, caracterizado por bloqueos mecánicos dolorosos de la rodilla, tiene un sustento anatómico basado en los fascículos poplíteo meniscales anteroinferior y posterosuperior, responsables de la estabilidad primaria de la esquina posterolateral meniscal. Con un cuadro clínico característico, usualmente con Resonancia Magnética sin hallazgos sugerentes de patología, la artroscopía juega un rol esencial en casos de alta sospecha, comprobando el diagnóstico al presentar hipermobilidad del cuerno posterior del menisco lateral. Método: Se presentan 9 casos resueltos, con hiperlaxitud posterolateral meniscal, y presentación clínica caracterizada por bloqueo articular de rodilla sin causa aparente. En todos los casos se realizó reparación de los fascículos poplíteo meniscales con suturas meniscales, con resolución completa de la sintomatología y sin complicaciones post quirúrgicas.

Conclusión El manejo del cuadro de menisco hipermóvil está basado por la sospecha clínica y el descarte de otras patologías como causa subyacente de la sintomatología. El manejo quirúrgico con suturas meniscales ha demostrado restaurar la biomecánica normal del compartimento posterolateral de la rodilla, logrando la resolución completa de los síntomas.

Nivel de evidencia IV.

Hypermobile Meniscus Syndrome, characterized by painful mechanical blockages of the knee, has an anatomical support based on the anteroinferior and posterosuperior poplíteomeniscal fascicles, responsible for the primary stability of the posterolateral meniscal corner. With a characteristic clinical presentation, usually with Magnetic Resonance without suggestive findings of pathology, arthroscopy plays an essential role in cases of high suspicion, checking the diagnosis by presenting hypermobility of the posterior horn of the lateral meniscus. Method: We present 9 resolved cases, with posterolateral meniscal hypermobility, and clinical presentation characterized by knee articular block without apparent cause. In all cases, poplíteomeniscal fascicles were repaired with meniscal sutures, with complete resolution of the symptoms and without post-surgical complications. 


\author{
Keywords \\ - knee \\ - hypermobile \\ lateral meniscus \\ - snapping knee
}

Conclusion The management of the hypermobile meniscus syndrome is based on clinical suspicion and the discarding of other pathologies as the underlying cause of the symptomatology. Surgical management with meniscal sutures has been shown to restore the normal biomechanics of the posterolateral compartment of the knee, achieving complete resolution of symptoms.

Level of evidence Case series IV.

\section{Introducción}

El Síndrome de Menisco Hipermóvil (SMH) o Subluxación Recurrente del Menisco Lateral (RSLM), corresponde a una entidad clínica caracterizada por episodios de bloqueo mecánico y doloroso de rodilla, sin inestabilidad aparente. En un comienzo, los episodios de bloqueos siempre ocurren durante la flexión mayor a $90^{\circ}$ de flexión. ${ }^{1}$ Hallazgos característicos en la artroscopia son la hipermovilidad del menisco lateral; subluxación anterior del menisco lateral con el uso de un palpador, en el que el margen periférico del segmento posterior del menisco lateral se mueve anteriormente más allá del cóndilo femoral lateral con la rodilla flectada a $90^{\circ}$; un fascículo poplíteo meniscal posterosuperior ( $\mathrm{SFPM}$ ) atenuado u obliterado, y un fascículo poplíteo meniscal antero inferior (iFPM) roto o atenuado. ${ }^{2}$

La unión del menisco medial con la cápsula articular es constante a lo largo de todo su borde periférico, sin tener fascículos separados para la porción superior e inferior del menisco, otorgándole de esa forma, una estabilidad intrínseca. En el caso del compartimento femoro-tibial lateral existe un hito constante en diversas preparaciones anatómicas, una discontinuidad de entre 1,2 y $1,5 \mathrm{cms}$ a través de la cual transcurre el tendón poplíteo, pasando de suporción intra-articular a suporción extra-articular, conocida como hiatopoplíteo (-Fig. 1).

Si bien las alteraciones alrededor del hiatopoplíteo han suscitado interés desde los primeros reportes de Higgins en $1895^{3}$ y posteriormente Cohn A.K. ${ }^{4}$ en la década de los 70 , su importancia clínica o las alteraciones en la biomecánica normal del menisco aún no se encuentran claramente definidas.

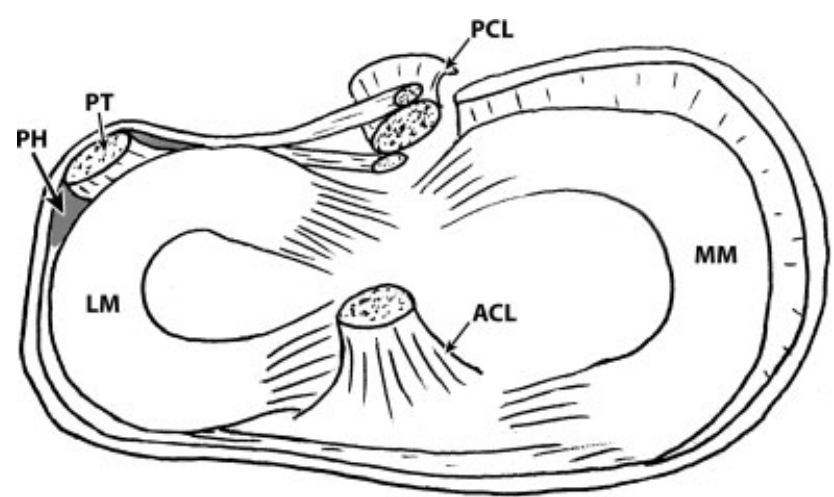

Fig. 1 Hiato poplíteo y su relación con otras estructuras intraarticulares en la rodilla. PT: tendón poplíteo; $P H$ : hiato poplíteo; LM: menisco lateral; $M M$ : menisco media; ACL: ligamento cruzado anterior; PCL: ligamento cruzado posterior.
Zappia M. ${ }^{5}$ describió los Fascículos Poplíteo meniscales (FPM) como estructuras meniscocapsulares posterolaterales que se originan en el cuerpo y cuerno posterior del menisco lateral, formando así el techo y piso del hiatopoplíteo. Cohn A. $\mathrm{K}^{4}{ }^{4}$ en su descripción anatómica describió que el hiatopoplíteo se encuentra delimitado por 2 fascículos: superior e inferior (-Fig. 2). Estas estructuras son las únicas que efectivamente se
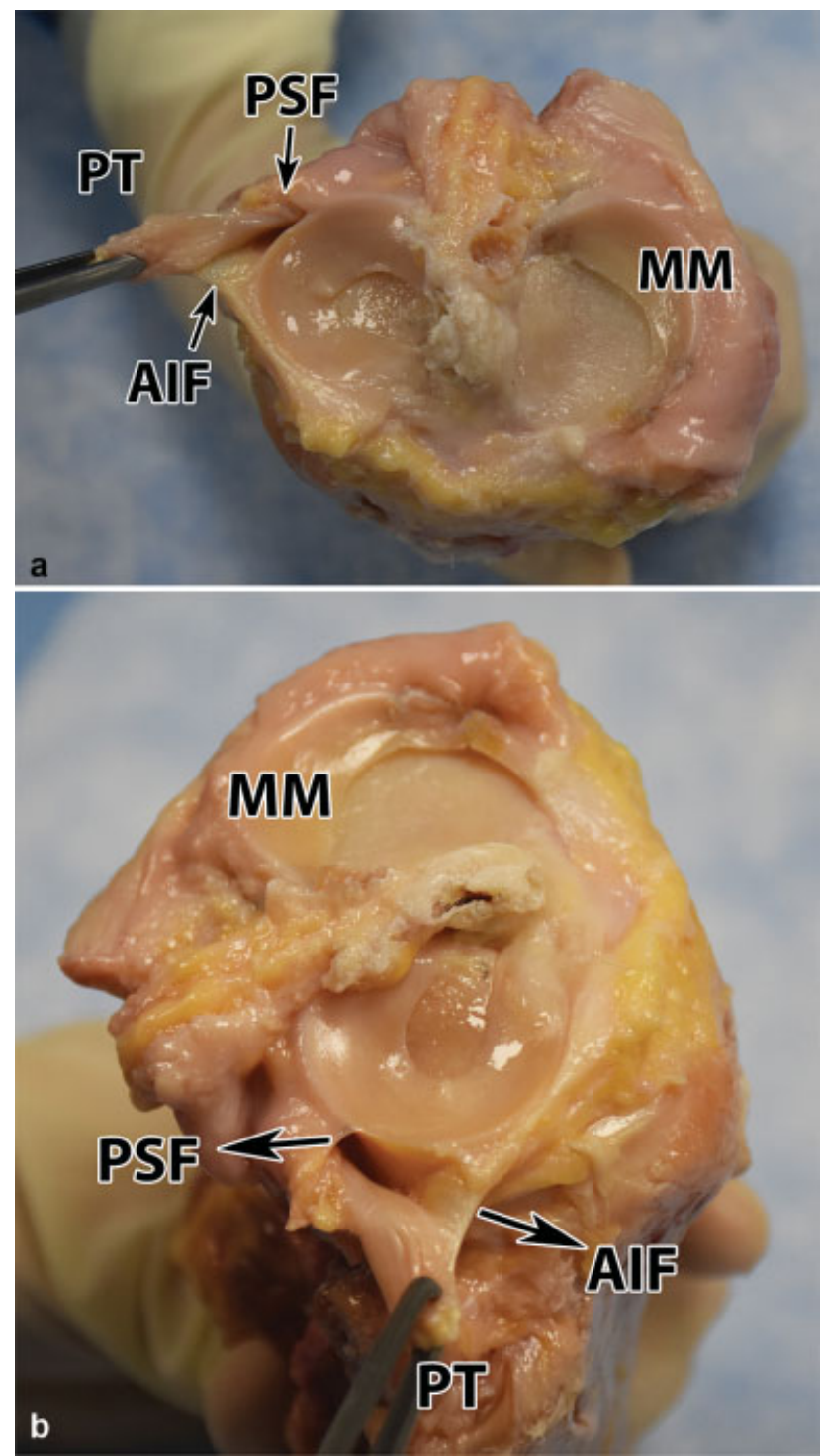

Fig. 2a y 2b Fascículos popliteomeniscales anteroinferior y posterosuperior en preparación anatómica. PT: tendón poplíteo; PSF: fascículo popliteomeniscal posterosuperior; AlF: fascículo popliteomeniscal anteroinferior; MM: menisco medial. 
adhieren a un borde meniscal definido: el fascículo inferior, llamado también "fascículo anteroinferior", o piso del hiato, está ubicado en situación más anterior; mientras que el fascículo superior, llamado también "fascículo posterosuperior", o techo del hiato, está en la zona más posterior. Publicaciones posteriores dan cuenta de un tercer fascículo, el fascículo "popliteomeniscal posteroinferior" ${ }^{6}$, que nace de la porción anterior del tendónpoplíteo, definiendo el límite anterior del hiato. El desarrollo embrionario de estos fascículos fue abordado por Sussmann y col., ${ }^{7}$ concluyendo que están conformados por 3 capas de tejido: capa de colágeno, capa vascular y capasinovial; y cuya función sería la de otorgar un aporte vascular a una porción periférica de menisco lateral.

La laxitudpropia de la unión menisco capsular, sumado a la discontinuidad de la misma en el hiatopoplíteo, hacen que el menisco lateral sea en la práctica más móvil que el medial. En ese sentido, Simonian, ${ }^{8}$ en un intento por cuantificar la estabilidad intrínseca del menisco externo, constató que ambos fascículos poplíteomeniscales contribuyen significativamente a la estabilidad del menisco, estableciéndose como estabilizadores primarios del desplazamiento anterior de la esquina posterolateral del menisco lateral en $90^{\circ}$ de flexión. De esa forma, se requiere una disrupción de los FPM para la inducción de un Síndrome de Menisco Hipermóvil.

El desafío inicial de ese tipo de lesiones radica en el diagnóstico. El cuadro clínico, dado principalmente por dolor lateral de rodilla asociado a episodios de bloqueo articular que pueden resolver espontáneamente o requerir de consulta médica, se correlaciona con una diversidad de diagnósticos diferenciales, tales como: Menisco discoideo sintomático, Cuerpo libre intraarticular, Síndrome de fricción de banda iliotibial, o Inestabilidad patelar, entre otros. LaPrade ${ }^{9}$ en una serie de casos de pacientes con Rotura de Fascículos Poplíteomeniscales describió el "Test de Figura en 4", mediante una maniobra de flexión, varo y rotación externa de rodilla, describiendo un test positivo como aquel que reproduce el bloqueo y/o dolor en la interlínea lateral de paciente. LaPrade describió dicho test como altamente sensible en el diagnóstico de este tipo de lesiones, presentándose en el 100\% de su serie. De esta forma, el "Test de Figura en 4" sería una herramienta fundamental en la orientación diagnóstica de pacientes con dolor en el compartimento lateral de la rodilla.

La carencia de correlato imagenológico resulta en un desafío adicional en el diagnóstico de Sindrome de Menisco Hipermóvil, con Resonancias Magnéticas habitualmente descritas como normales. No obstante, este punto sigue siendo controversial. Simonian, ${ }^{10}$ en un reporte de casos, demostró que las lesiones de PMF eran detectables mediante estudios por Resonancia Magnética; hallazgo que fue posteriormente confirmado por Johnson y De Smet, ${ }^{11}$ en un estudio retrospectivo, donde en 64 de 66 rodillas pudieron detectar ambos fascículos en resonancias de rutina. Sakai, ${ }^{12}$ en un estudio prospectivo con 34 voluntarios sanos, encontró que en un $88 \%$ y un $94 \%$ de ellos se encontraban presentes los SPMF e iPMF respectivamente, y estableció que mejores resultados pueden ser obtenidos mediante resonancias con cortes coronales oblícuos a $45^{\circ}$.

A pesar de los avances en el conocimiento de este tipo de lesiones, la evaluación mediante artroscopia sigue siendoen la actualidad el gold standard para el diagnóstico de certeza, puesto que permite una visualización directa de los fascículos poplíteo-meniscales y la evaluación de la movilidad del menisco lateral. ${ }^{13}$

Existen múltiples técnicas quirúrgicas publicadas para el tratamiento de este tipo de patología: meniscectomía, ${ }^{14}$ reduccióntérmica meniscal, ${ }^{15,16}$ reparación con sutura meniscal en forma abierta ${ }^{9}$ y artroscópica, ${ }^{17,18}$ además reconstrucción de fascículos poplíteomeniscales con utilización de injerto. ${ }^{1}$

Dado lo poco frecuente del Síndrome de Menisco Hipermóvil, el propósito de esta publicación es describir nuestra experiencia en el diagnóstico y tratamiento de este tipo de lesiones dentro de un grupo de traumatólogos especialistas en rodilla, en relación a 9 casos clínicos. Esto, fundamentado con una revisión bibliográfica de lo publicado hasta la fecha.

\section{Materiales y Métodos}

Serie de nueve casos clínicos evaluados y tratados entre el año 2012 y 2017, resueltos por un equipo de traumatólogos con experiencia en cirugía de alta complejidad de rodilla.

Se incluyeron todos los casos con diagnóstico comprobado de menisco lateral hipermóvil mediante el uso de artroscopia, y en los que se efectuó reparación de los fascículos poplíteomeniscales con suturas meniscales. Los casos resueltos con técnica "todo dentro" y aquellos con técnica "dentro-fuera" fueron incluidos en el mismo grupo de análisis. Se excluyeron los casos asintomáticos, como también aquellos que presentaron patología concomitante de rodilla: rotura meniscal, lesiones ligamentarias, y artrosis; presencia de meniscodiscoídeo, y aquellos sin diagnóstico confirmado de síndrome de menisco hipermóvil. La indicación quirúrgica de reparación de los fascículos se basó en criterios clínicos y artroscópicos, tales como la presencia de síntomas dados por dolor y/o episodios de bloqueo articular; el desplazamiento del segmento posterior del menisco lateral mayor a un 50\% hacia anterior en relación al diámetro anteroposterior del platillo tibial externo (traslación anterior igual o mayor al centro del platillo tibial) y la subluxación del menisco lateral tras realizar una maniobra de tracción con un palpador artroscópico (-Fig. 3 y 4 ).

Se presentan las características demográficas de cada paciente, así como una breve reseña sobre su presentación clínica, con especial énfasis en la sintomatología principal y hallazgos en la resonancia magnética para cada caso (- Tabla $\mathbf{1}$ ). Cada caso describe la técnica quirúrgica utilizada, de manera de evaluar la evolución que ha tenido este tipo de cirugía en un corto plazo de tiempo, tomando en cuenta la experiencia y avances tanto en conocimientos como en instrumental quirúrgico.

Independientemente de la técnica de fijación meniscal utilizada, se siguieron principios quirúrgicos dirigidos a reproducir los vectores de fuerza de los fascículos poplíteomeniscales (-Fig. 5 y $\mathbf{6}$ ). De esta forma, se posicionó el primer punto de sutura a través de la hoja superior de menisco lateral, en posición posterior a tendón poplíteo (fascículo 


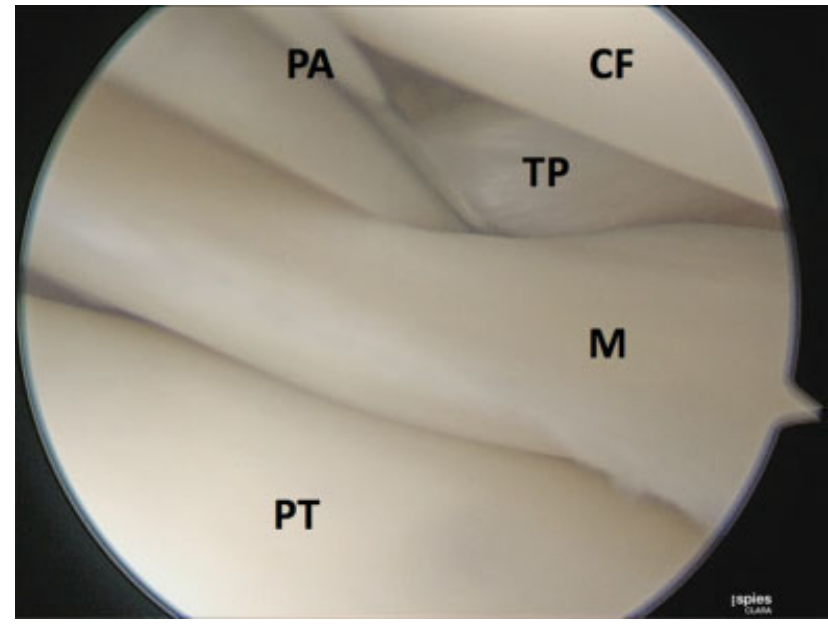

Fig. 3 Evaluación hiato poplíteo con palpador artroscópico. TP: tendón poplíteo; M: menisco; PA: palpador artroscópico; CF: cóndilo femoral; PT: platillo tibial.

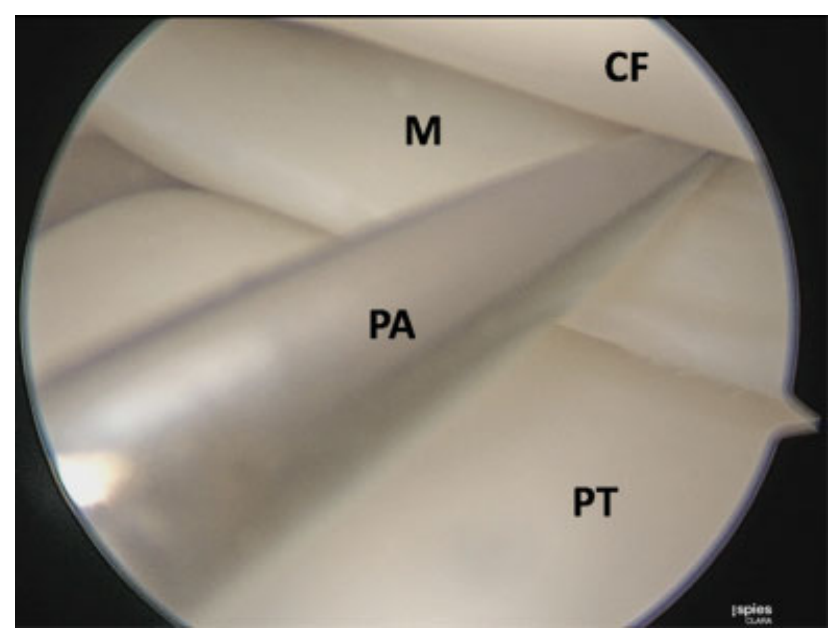

Fig. 4 Luxación de cuerno posterior meniscal a zona anterior a cóndilo femoral. M: menisco; PA: palpador artroscópico; CF: cóndilo femoral; PT: platillo tibial.

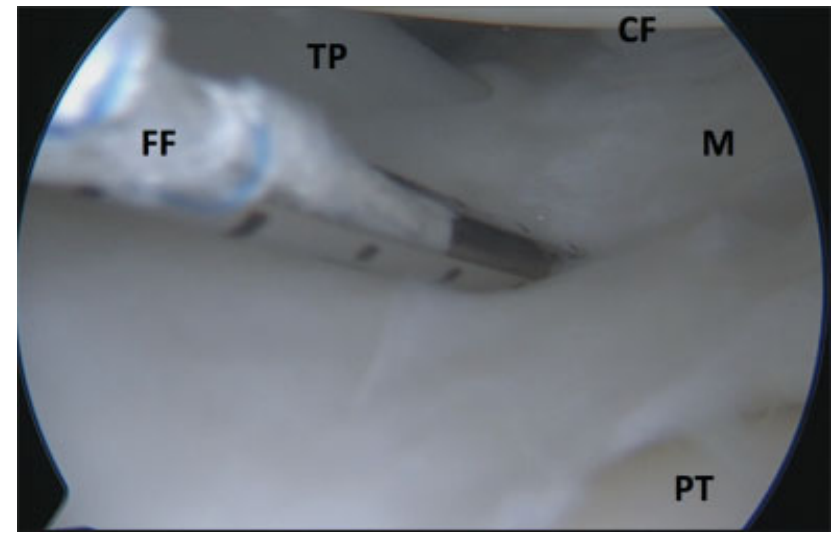

Fig. 5 Instalación de ancla FastFix 360 (Smith \& Nephew Endoscopy, Andover, MA) a través de hoja superior de menisco lateral, en posición posterior a tendón poplíteo. TP: tendón poplíteo; M: menisco; CF: cóndilo femoral; PT: platillo tibial; FF: dispositivo FastFix 360.

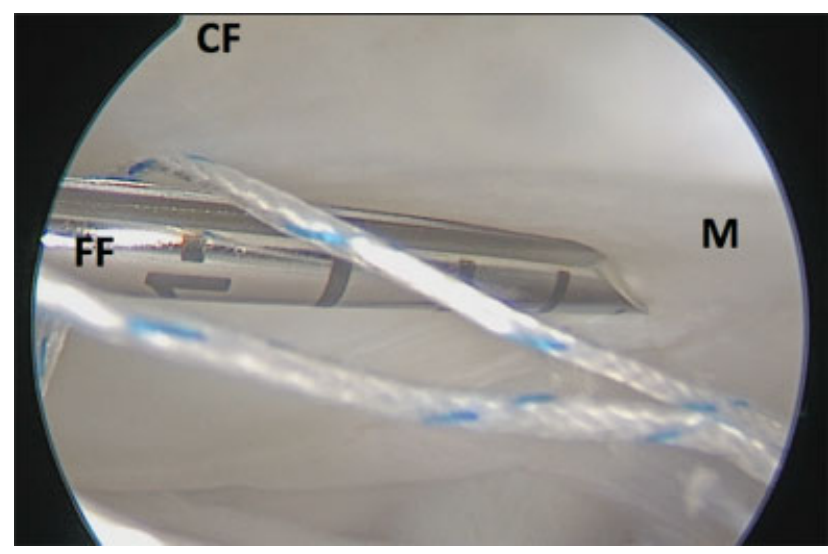

Fig. 6 Instalación de ancla FastFix 360 (Smith \& Nephew Endoscopy Andover, MA) a través de hoja superior de menisco lateral, en posición posterior a tendón poplíteo. M: menisco; CF: cóndilo femoral; FF: dispositivo FastFix 360.

Tabla 1 Datos Demográficos de 9 Pacientes Con Menisco Lateral Hipermóvil Sintomático

\begin{tabular}{|l|l|l|l|l|l|l|}
\hline Paciente & Género & Edad & Deporte & Síntomas predominantes & $\begin{array}{l}\text { Hallazgos en } \\
\text { Resonancia Magnética }\end{array}$ & $\begin{array}{l}\text { Técnica de } \\
\text { reparación }\end{array}$ \\
\hline 1 & Hombre & 17 & Parkour & $\begin{array}{l}\text { Bloqueo mecánico en } \\
\text { flexión máxima }\end{array}$ & Dentro-fuera \\
\hline 2 & Mujer & 18 & No realiza & Bloqueo mecánico & Normal & Todo adentro \\
\hline 3 & Mujer & 14 & No realiza & $\begin{array}{l}\text { Bloqueo mecánico, dolor } \\
\text { posterolateral }\end{array}$ & Dentro-fuera \\
\hline 4 & Hombre & 14 & Rugby & Bloqueo mecánico & $\begin{array}{l}\text { Desinserción capsular } \\
\text { de menisco lateral }\end{array}$ & Todo adentro \\
\hline 5 & Mujer & 15 & Fútbol & Bloqueo mecánico & Normal & Dentro-fuera \\
\hline 6 & Hombre & 15 & Basquetbol & $\begin{array}{l}\text { Bloqueo mecánico, dolor posterolateral, } \\
\text { chasquido de rodilla }\end{array}$ & Normal & Todo adentro \\
\hline 7 & Mujer & 13 & Gimnasia & Bloqueo mecánico, dolor posterolateral & Normal & Todo adentro \\
\hline 8 & Mujer & 14 & Gimnasia & $\begin{array}{l}\text { Bloqueo mecánico, dolor posterolateral, } \\
\text { chasquido de rodilla }\end{array}$ & $\begin{array}{l}\text { Desinserción capsular } \\
\text { de menisco lateral }\end{array}$ & Todo adentro \\
\hline 9 & Mujer & 16 & Gimnasia & $\begin{array}{l}\text { Bloqueo mecánico en } \\
\text { flexión máxima }\end{array}$ & Normal & Todo adentro \\
\hline
\end{tabular}




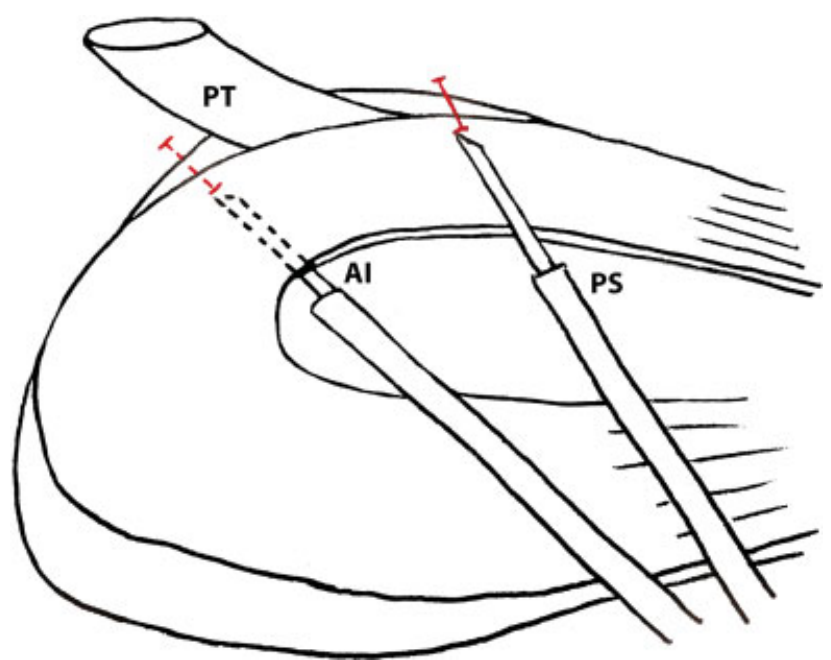

Fig. 7 Esquema de procedimiento artroscópico. Reparación de hiato poplíteo con 2 suturas FastFix 360 (Smith \& Nephew Endoscopy, Andover, MA). Instalación de 1 sutura en posición de fascículo anteroinferior y 1 sutura en posición de fascículo posterosuperior. PT: tendón poplíteo; Al: FastFix 360 anteroinferior; PS: FastFix360 posterosuperior.

posterosuperior); y el segundo punto de sutura a través de la hoja inferior de menisco lateral, en posición anterior al tendón poplíteo (fascículo anteroinferior) (-Fig. 7).

\section{Resultados}

De los nueve casos, seis correspondieron a mujeres (67\%) y tres a hombres (33\%), con una mediana de edad de 15 años, ${ }^{15-19}$ siete de ellos deportistas. En el 100\% de los casos el bloqueo mecánico de rodilla en flexión fue el síntoma predominante. El dolor y chasquido posterolateral de rodilla también fueron formas de presentación, manifestándose en el 44\% y 22\% de los casos respectivamente. Sólo dos resonancias magnéticas arrojaron resultados positivos, evidenciados por edema en relación al tendón poplíteo a nivel del hiato y de inserción capsular del menisco lateral.

La totalidad de los pacientes fueron sometidos a reparación de los fascículos poplíteo meniscales, mediante fijación meniscal artroscópica del menisco externo a la cápsula posterior con suturas meniscales. En tres pacientes se realizó la reparación con técnica dentro-fuera y en seis con técnica todo-dentro.

Todos los pacientes evolucionaron con resolución completa de los síntomas y no presentaron complicaciones post quirúrgicas inmediatas. Asimismo, se realizó seguimiento de la evolución clínica de cada caso por al menos 1 año posterior a la fecha de la intervención, y no se evidenciaron recidivas ni reoperaciones.

\section{Discusión}

El Síndrome de Menisco Hipermóvil es una entidad clínica que se caracteriza por dolor y episodios de bloqueo articular durante la flexión profunda de rodilla. Fisiológicamente, esta sintomatología encuentra su sustrato en un "movimiento paradójico" del menisco externo, en que se observa una traslación anterior del cuerno posterior durante la flexión de rodilla, y traslación posterior del mismo segmento meniscal durante la extensión. ${ }^{20}$ De acuerdo a los hallazgos descritos por Vadi y col., ${ }^{21}$ en un menisco lateral con fascículos poplíteo meniscales intactos, ese movimiento durante la movilización articular de rodilla es inverso, con traslación posterior del cuerno posterior durante la flexión y traslación anterior durante la extensión.

De esa forma, la disrupción o ausencia de los Fascículos Poplíteo meniscales es la piedra angular para el desarrollo de un Síndrome de Menisco Hipermóvil. Esta alteración de los elementos estabilizadores posterolaterales del menisco externo ha sido atribuida a un defecto congénito que determina la ausencia de estos fascículos, o bien, a una lesión secundaria a un traumatismo sobre el Complejo Cápsulo-meniscal Posterior (ligamentos meniscotibiales y/o poplíteomeniscales). ${ }^{22}$ Esto último fue constatado por LaPrade y cols, ${ }^{19}$ en un estudio prospectivo que incluyó 30 rodillas de pacientes con lesiones del Complejo posterolateral. En ellos, se realizó evaluación artroscópica donde se evidenció que todas (las 30 rodillas) presentaron lesiones de algún componente del Complejo Poplíteo: 83\% del fascículo anteroinferior, $80 \%$ del ligamento coronario (ligamentomeniscotibial), y 73\% del fascículo posterosuperior.

Dado la excesiva excursión del menisco lateral que resulta de la ausencia o insuficiencia de los Fascículos Poplíteomeniscales, las escasas publicaciones acerca de este tipo de patología reportan una improbable posibilidad de éxito con el tratamiento conservador. ${ }^{22}$ Es así como se ha planteado que los pacientes podrían beneficiarse con un procedimiento quirúrgico simple, dirigido a la fijación meniscal artroscópica a la cápsula posterior. ${ }^{18}$ Esto puede realizarse mediante una técnica todo dentro o dentro fuera según las preferencias del cirujano y el instrumental disponible.

El principal hallazgo de la propuesta de tratamiento publicada en esta serie de casos radica en la factibilidad de cicatrización que tiene este segmento meniscal, tanto por ser una zona vascularizada del menisco, ${ }^{23-25}$ como por la función de vascularización que podrían cumplir los fascículos popliteomeniscales. ${ }^{7} \mathrm{Si}$ bien, los primeros casos fueron resueltos mediante técnica dentro fuera, destacamos la facilidad técnica de realizar una sutura con técnica todo dentro con el instrumental moderno. Al realizar este tipo de reparación, se disminuye la longitud efectiva del hiatopoplíteo, acercándolo a sus valores anatómicos normales. Además, se recrean los vectores de fuerza de cada uno de los fascículos popliteomeniscales en forma separada, orientando las suturas desde el borde superior e inferior meniscal respectivamente para cada fascículo según su ubicación en relación al tendón poplíteo. Arendt y cols. ${ }^{26}$ reportaron 11 pacientes y 12 rodillas con diagnóstico previo de luxación recidivantepatelar, con tratamientos fallidos, y con diagnóstico de Síndrome de Menisco Hipermóvil, confirmado con artroscopia. Todos los casos fueron resueltos con suturas dentro fuera, sin complicaciones ni recurrencias. De esta forma, concluyeron que la reparación de los Fascículos Poplíteomeniscales es esencial para la estabilización meniscal. Steinbacher y cols. ${ }^{22}$ reportan recientemente un estudio retrospectivo con 46 casos 
en jugadores de fútbol, con un seguimiento a 48 meses, tratados con suturas todo dentro, con uno (5\%), dos (59\%), tres (22\%) y cuatro (2\%) nudos de sutura en diferentes configuraciones. El retorno deportivo pre-lesional se logró en sólo 56\% de los casos, y se lograron buenos resultados funcionales, objetivados con los scores IKDC y Tegner. Nuestro reporte considera pacientes más jóvenes (15 años), en los cuales el Síndrome de Menisco Hipermóvil es más prevalente. Además, consideramos que la anatomía debe ser tomada en cuenta cuando se realiza la fijación meniscal, siendo esencial el restablecimiento de los vectores de fuerza. En este sentido, sólo dos nudos de suturas serían suficientes para estabilizar el menisco lateral.

Hubo algunas limitaciones en este estudio. En primer lugar, corresponde a una serie de casos sin grupo de control y, dado la baja incidencia de este tipo de patología, con un número pequeño de pacientes, lo que es concordante con publicaciones anteriores sobre el mismo tema. En segundo lugar, si bien proponemos una estrategia de tratamiento que ha evidenciado muy buenos a excelentes resultados en la literatura, aún no han sido reportados estudios biomecánicos que demuestren que esta estrategia es superior a otras que han sido publicadas al respecto como, por ejemplo, la reconstrucción de los fascículos poplíteomeniscales.

\section{Conclusión}

En conclusión, este tipo de patología refuerza la idea de que la historia clínica y el examen físico son herramientas fundamentales al realizar un diagnóstico adecuado. Creemos que la resonancia magnética juega un rol importante al descartar otro origen para la sintomatología, sin embargo, ante la posibilidad de un examen imagenológico sin hallazgos patológicos, y con la certeza de una historia clínica concordante con esta patología, se debe realizar una inspección artroscópica y eventualmente tratamiento quirúrgico. La fijación meniscal a la cápsula posterior ha mostrado buenos a excelentes resultados clínicos y funcionales.

Conflicto de Intereses

Lo autor del trabajo declara no tener ningún conflicto de intereses.

\section{Bibliografía}

1 Suganuma J, Inoue $\mathrm{Y}$, Tani $\mathrm{H}$, Sugiki T, Sassa T, Shibata R Reconstruction of the Popliteomeniscal Fascicles for Treatment of Recurrent Subluxation of the Lateral Meniscus. Arthrosc Tech 2017;6(02):e283-e290

2 Ahn JH, Lee SH, Kim KI, Nam J. Arthroscopic meniscus repair for recurrent subluxation of the lateral meniscus. Knee Surg Sports TraumatolArthrosc 2018;26(03):787-792

3 Higgins H. The Geniculate Articular Surfaces of the Femur and Tibia. J AnatPhysiol 1895;29(Pt 4):574-582.1, 1

4 Cohn AK, Mains DB. Popliteal hiatus of the lateral meniscus. Anatomy and measurement at dissection of 10 specimens. Am J Sports Med 1979;7(04):221-226

5 Zappia M, Reginelli A, Chianca V, et al. MRI of popliteo-meniscal fasciculi of the knee: a pictorial review. Acta Biomed 2018;89 (1-S):7-17
6 Terry GC, LaPrade RF. The posterolateral aspect of the knee. Anatomy and surgical approach. Am J Sports Med 1996;24(06):732-739

7 Sussmann PS, Simonian PT, Wickiewicz TL, Warren RF. Development of the popliteomeniscal fasciculi in the fetal human knee joint. Arthroscopy 2001;17(01):14-18

8 Simonian PT, Sussmann PS, van Trommel M, Wickiewicz TL, Warren RF. Popliteomeniscal fasciculi and lateral meniscal stability. Am J Sports Med 1997;25(06):849-853

9 LaPrade RF, Konowalchuk BK. Popliteomeniscal fascicle tears causing symptomatic lateral compartment knee pain: diagnosis by the figure- 4 test and treatment by open repair. Am J Sports Med 2005;33(08):1231-1236

10 Simonian PT, Sussmann PS, Wickiewicz TL, et al. Popliteomeniscal fasciculi and the unstable lateral meniscus: clinical correlation and magnetic resonance diagnosis. Arthroscopy 1997;13(05): 590-596

11 De Smet AA, Asinger DA, Johnson RL. Abnormal superior popliteomeniscal fascicle and posterior pericapsular edema: indirect MR imaging signs of a lateral meniscal tear. AJR Am J Roentgenol 2001;176(01):63-66

12 Sakai H, Sasho T, Wada Y, et al. MRI of the popliteomeniscal fasciculi. AJR Am J Roentgenol 2006;186(02):460-466

13 Camarillo M, Johnson DL. Popliteomeniscal fascicle tears. Orthopedics 2014;37(03):187-190

14 Kimura M, Shirakura K, Hasegawa A, Kobayashi Y, Udagawa E. Anatomy and pathophysiology of the popliteal tendon area in the lateral meniscus: 1. Arthroscopic and anatomical investigation. Arthroscopy 1992;8(04):419-423

15 Higuchi H, Kimura M, Kobayashi A, Hatayama K, Takagishi K. A novel treatment of hypermobile lateral meniscus with monopolar radiofrequency energy. Arthroscopy 2004;20(Suppl 2):1-5

16 Ohtoshi K, Kimura M, Kobayashi Y, Higuchi H, Kikuchi S. Arthroscopic thermal shrinkage for hypermobile lateral meniscus. Am J Sports Med 2004;32(05):1297-1301

17 Shin HK, Lee HS, Lee YK, Bae KC, Cho CH, Lee KJ. Popliteomeniscal fascicle tear: diagnosis and operative technique. Arthrosc Tech 2012;1(01):e101-e106

18 Van Steyn MO, Mariscalco MW, Pedroza AD, Smerek J, Kaeding CC, Flanigan DC. The hypermobile lateral meniscus: a retrospective review of presentation, imaging, treatment, and results. Knee Surg Sports TraumatolArthrosc 2016;24(05):1555-1559

19 LaPrade RF. Arthroscopic evaluation of the lateral compartment of knees with grade 3 posterolateral knee complex injuries. Am J Sports Med 1997;25(05):596-602

20 Kamiya T, Suzuki T, Otsubo $\mathrm{H}$, et al. Midterm outcomes after arthroscopic surgery for hypermobile lateral meniscus in adults: Restriction of paradoxical motion. J Orthop Sci 2018;23(06): 1000-1004

21 Vedi V, Williams A, Tennant SJ, Spouse E, Hunt DM, Gedroyc WM. Meniscal movement. An in-vivo study using dynamic MRI. J Bone Joint Surg Br 1999;81(01):37-41

22 Steinbacher G, Alentorn-Geli E, Alvarado-Calderón M, Barastegui D, Álvarez-Díaz P, Cugat R. Meniscal fixation is a successful treatment for hypermobile lateral meniscus in soccer players. Knee Surg Sports TraumatolArthrosc 2019;27(02):354-360

23 Arnoczky SP, Warren RF. Microvasculature of the human meniscus. Am J Sports Med 1982;10(02):90-95

24 Arnoczky SP, Warren RF. The microvasculature of the meniscus and its response to injury. An experimental study in the dog. Am J Sports Med 1983;11(03):131-141

25 Henning CE, Lynch MA, Clark JR. Vascularity for healing of meniscus repairs. Arthroscopy 1987;3(01):13-18

26 Arendt EA, Fontboté CA, Rohr SR. Displacing lateral meniscus masquerading as patella dislocation. Knee Surg Sports TraumatolArthrosc 2014;22(10):2315-2319 